\title{
Wissenschaftsethik für Mediziner - Lehren aus der Causa zu Guttenberg?
}

\author{
Bettina Schöne-Seifert
}

Online publiziert: 19. April 2011

(C) Springer-Verlag 2011

Ein Bundesminister, dessen Dissertationsschrift von abgeschriebenen Passagen nur so strotzt. Kabinettskollegen, die ihm die Lüge vom fehlenden Vorsatz - Hand aufs Herz bereitwillig abkaufen. Eine universitäre Kommission, die zunächst selektiv untersucht. Ein einflussreicher Teil der medialen Öffentlichkeit, der den Beschuldigten zum Opfer politischer und sozialer Neider stilisiert. Das alles ist ärgerlich, peinlich, bedrückend. Aber vielleicht dennoch kein Gegenstand für das Editorial einer medizinethischen Fachzeitschrift.

Hierzu wird die „Affäre Guttenberg“ erst dadurch, dass bei dieser Gelegenheit so viele Menschen eine bestürzende Einstellung zum Abschreibungsbetrug zu erkennen geben: Die einen sehen Plagiate als lässliche Kleinsünde, als etwas, was ,wir doch alle mal gemacht haben" - alle bis auf die unsympathischen moralisierenden Streber. Andere trauen der Wissenschaft überhaupt nicht recht über den Weg. Sie finden das Abkupfern juristischer Phrasen vergleichsweise harmlos, im Vergleich nämlich zu Klonskandalen, der Veruntreuung von Forschungsmillionen oder dem Türken medizinischer Studien, die Einfluss auf die Behandlung von Patienten haben. Beides, das Verharmlosen wie der Generalverdacht sind alarmierende Befunde. Das ändert sich auch nicht dadurch, dass es ausgerechnet Doktoranden (ca.14.000) und Hochschullehrer (etwa 1.000) waren, die über offene Briefe mit Massenunterschriften wohl wesentlich zum Rücktritt des Verteidigungsministers beigetragen haben. Diese Kampagnen werden viele Guttenberg-Apologeten ohnehin nur als lächerlich empfinden, als naiv in der Wahrnehmung oder strategisch in der Motivation. Zudem haben die großen Wissenschaftsorganisationen zu leise, zu spät oder auch gar nicht reagiert. Wohl hat der Ex-Präsident der Deutschen Forschungsgemeinschaft, Ernst-Ludwig Winnacker, als einer der ersten klare Worte gesprochen, haben auch die amtierenden Präsidenten etwa der Leopoldina, der DFG oder des Hochschulrates den Umgang mit dem Plagiator schon vor dessen Rücktritt deutlich kritisiert. Aber warum gab es hier keine konzertierte Aktion? Keine schriftliche Stellungnahme, um die man doch auch sonst ad hoc nicht verlegen ist?

B. Schöne-Seifert $(\bowtie)$

Institut für Ethik, Geschichte \& Theorie der Medizin, Universität Münster,

Von-Esmarch-Str. 62, 48149 Münster, Deutschland

E-Mail: bseifert@uni-muenster.de 
Was also ist zu tun? Offenbar ein Tätigwerden auf drei Ebenen:

(1) Innerhalb der Wissenschaft selbst müssen die zukünftigen und gegenwärtigen Akteure noch besser als bisher für Probleme des Forschungsbetrugs in seinen vielfältigen Varianten sensibilisiert und vorbereitet werden. Sie müssen verstehen lernen, dass wissenschaftliche Integrität eine Alles-oder-Nichts-Tugend ist, das Fundament für interne Kooperation und externes Vertrauen, und damit für wissenschaftlichen Fortschritt.

Insbesondere für die Medizin gilt: Die üblichen Doppelrollen als Student und Promovend, als Ärztin und Forscherin liefern keine Entschuldigung für auch nur kleine Abstriche an Ehrlichkeit. Auch nicht der enorme Zeit- und Konkurrenzdruck, unter dem hier besonders oft gearbeitet wird. Ebenso wenig der hohe Grad an kollegialer Interessenvernetzung: Gefälligkeiten und Vorteilsnahmen als Gutachter oder Ko-Autor, das Aufblähen von Publikationslisten, Selbstplagiate und Zitierkartelle zur Erhöhung von impact-Faktoren - alles bekannte Phänomene - müssen identifiziert und bekämpft werden.

(2) Im Wissenschafts-Management sollten (weitere) strukturelle Weichenstellungen zur Beförderung ehrlichen Arbeitens gestellt werden. Dazu gehören auch härtere Sanktionen für Regelverstöße und ein entschiedenes Vorgehen gegen die deutsche Promotions-Inflation: „Die Universitäten sollten diese Titelschwemme eindämmen“, schreibt zu Recht Martin Spiewak, und nur, ,jene promovieren lassen, die es in die Wissenschaft zieht. Das gilt zu allererst für Mediziner, deren Doktorarbeit oft den Namen nicht verdient" ([3], S. 40). Diese Einsicht ist nicht neu.

Insbesondere für die Medizin gilt: Schon die DFG-Empfehlungen von 1998 zur Verhinderung und Handhabung von Forschungsbetrug sprechen dieses Problem an [1]. ,Feierabendforschung“ wie sie zahlreichen (nicht allen) Mediziner-Promotionen zugrunde liegt, führt leicht zu Unregelmäßigkeiten, zu wissenschaftlich Überflüssigem - und zu ungerechten Beurteilungen im Vergleich zu anderen Fächern. Von dem Doppelstandard, mit dem medizinische und nicht-medizinische Dissertationen fast durchgehend beurteilt werden müssen, können gerade die Vertreter der Fächer Geschichte, Theorie und Ethik in der Medizin ein leidiges Lied singen.

Wissenschaftsrat und Kultusministerkonferenz haben in den letzten Jahren deutlich auf eine Trennung von Studien- und Promotionsphase auch bei Medizinern gedrungen und die anglo-amerikanische Unterscheidung zwischen $\mathrm{MD}$ und $\mathrm{PhD}$ auch für Deutschland empfohlen. Es wird Zeit, an die Umsetzung zu gehen.

(3) In die nicht-akademische Öffentlichkeit müssen Natur und Wert wissenschaftlicher Ehrlichkeit sowie die ihrem Schutz dienenden Maßnahmen besser kommuniziert werden. Mit den Worten Winnackers: „Die Gesellschaft weiß vielleicht insgesamt noch zu wenig von den Regeln der Wissenschaftskultur und schätzt zu wenig, wie wichtig diese Regeln dafür sind, dass mit Steuergeldern auch wirklich gute Wissenschaft geleistet wird" [4]. Insbesondere für die Medizin gilt: Die Anerkennung für ,wirklich gute Wissenschaft“ und die Mühen, die sie fordert, muss wachsen. Ebenso aber auch die Anerkennung für gut ausgebildete Mediziner ohne Doktortitel - bei Arbeitgebern wie Patienten.

Die ethischen Fragen, um die es hier geht, sind zu wichtig, als dass sie weiterhin vernachlässigt werden dürften. Ulrich Schnabels dringender Appell, dass wissenschaftliche Exzellenz nicht ,zum moralischen Nulltarif“ zu haben sei ([2], S.33), ist nur allzu berechtigt. Sie ist auch nicht zum wissenschaftsethischen Nulltarif zu haben. 


\section{Literatur}

1. Deutsche Forschungsgemeinschaft (1998) Sicherung guter wissenschaftlicher Praxis. http://www.dfg. de/download/pdf/dfg_im_profil/reden_stellungnahmen/download/empfehlung_wiss_praxis_0198.pdf. Zugegriffen: 26. März 2011

2. Schnabel U (2011) Die Titelverteidiger. DIE ZEIT (03. März): 33-34

3. Spiewak M (2011) Ein Frage der Ehre. DIE ZEIT (24. Februar): 39-40

4. Winnacker EL (2011) Für immer am Pranger. Interview mit C. Schwägerl. DER SPIEGEL (28. Februar): 27 